\title{
Programmed Cell Death in Plants
}

\author{
Ko Shimamoto*, Akira Takahashi*, Kenji Henmi*, Eiichiro Ono*, Satoru Hatakeyama*, \\ Megumi Iwano** and Tsutomu Kawasaki* \\ * Laboratory of Plant Molecular Genetics and ${ }^{* *}$ Graduate Schhol of Biological Sciences, Nara Institute of Science and Technology, \\ 8916-5 Takayama, Ikoma 630-0101, Japan \\ (Correspondence; Ko Shimamoto, Fax, 81-743-72-5509; E-mail,
}

\begin{abstract}
There is increasing evidence for occurrence of programmed cell death (PCD) in plant development, plant-microbe interaction and cells under a variety of stresses. Recent studies on PCD in plants indicate that various features of apoptosis in mammals are shared with plant PCD: there is evidence for DNA fragmentation, oligonucleosomal DNA laddering, morphological changes in plant cells. These studies suggest that PCD plays an important role in the life of plants as in animals. Despite the wide occurrence of PCD in plants, signaling and components of the machinery for PCD are largely unknown. We recently identified the Rac family of the small GTP-binding protein as a key regulator of PCD in plants. Also, the analysis of lesion mimic mutants of rice indicates that some mutants have biochemical alterations in early steps of signaling in disease resistance. The major challenge in the study of plant PCD in the near future is the identification of signaling molecules and components of machinery involved in plant PCD. This will enable us to better understand this important cellular process of plants.
\end{abstract}

\section{Introduction}

Programmed cell death (PCD) occurs during development and resistance responses to pathogens in plants [1-3]. Although the phenomenon has been known for a long time in plants, only recently PCD in plants has received attention mainly due to the rapid progress of studies of apoptosis in animals $[4,5]$. In this review, we discuss our current understanding of plant PCD.

\section{PCD in plant development}

PCD occurs in a number of tissues during development of plants. Some examples are described below.

Aleurone cells: Aleurone cells, the outmost layers of grass seeds, undergo PCD during germination [6]. Aleurone cells are terminally differentiated cells and during germination they release various hydrolytic enzymes to digest endosperm to provide nutrients necessary for germination of embryos. After completion of germination aleurone cells are completely dead. In barley aleurone cells, it was shown that nuclear DNA is cleaved into oligonucleosome-sized fragments and that this DNA fragmentation is stimulated by gibberelin (GA) and blocked by abscisic acid (ABA) [6].

Tracheary elements: Tracheary elements (TEs) are dead cells in the vascular tissues and their differentiation involves autolysis [7]. Autolysis begins with lobing of the cytoplasm and nuclei, their shrinkage and condensation completes with degradation of nuclear DNA. PCD that occurs during differentiation of TEs has been extensively studied by culturing mechanically isolated Zinnia elegans mesophyll cells under defined conditions [7]. This experimental system has provided much of our knowledge on plant PCD [7].

Roots: Examples of PCD are found during develop- ment of roots. Aerenchyma cells are formed by PCD to produce internal air spaces to facilitate efficient oxygen supply in the soil [8]. Another form of PCD that occurs in roots is found in root cap cells [9]. Root cap cells are produced from the meristem and sloughed from the root while new cells are continuously generated.

Leaf: Leaf senescence is a well known example of cell death in plant development [10]. When old leaves senesce active transcription of new genes occurs, suggesting that senescence is an active process and some genes encode hydrolase such as proteases [10]. Although morphological changes of cells and DNA cleavage typically observed in cells undergoing PCD have not been clearly demonstrated in senescing leaves yet, a number of physiological studies strongly suggest that it involves PCD.

Flower: Two examples of PCD have been recently demonstrated in flowers. In pea flowers, the carpel undergoes PCD when pollination is prevented by emasculation. This senescence of the carpel is stimulated by ethylene and inhibited by treatment with GA. In dying carpels, oligonucleosomal DNA fragmentaion has been observed [11]. Another example of PCD in flower development is elimination of sex organ in unisexual flowers. For instance, in maize stamen primordia abort early in development of female flowers [12].

Many examples of PCD during normal development of plants described above strongly suggest that plants employ PCD to eliminate some cells or generate specialized cells such as TEs in development. However, at the moment little is known on molecular mechanisms regulating the PCD in plant development. Furthermore, whether there are multiple pathways for various PCDs observed during development remains to be studied in the future. 


\section{Plant PCD induced by external stimuli}

A large number of external stimuli have been recently shown to induce PCD in plant cells as in mammalian cells. Evidence for PCD is one or more of characteristics; they are morphological changes of cells such as shrinkage of cells, condensation of nucleus and chromatin, positive TUNEL staining and oligonucleosomal laddering of nuclear DNA.

Phytotoxin The host specific AAL toxin of a pathogenic fungus, Alternalia alternata $\mathrm{f}$ sp lycopersici, was shown to cause laddering of nuclear DNA in tomato seedlings and protoplasts [9]. $\mathbf{H}_{\mathbf{2}} \mathbf{O}_{\mathbf{2}}$ that is implicated in hypersensitive cell death during resistance reactions to pathogens has been shown to induce PCD in suspension cultured plant cells [13], however, no clear oligonucleosomal DNA laddering has not been observed. $\mathbf{K C N}$ was shown to cause DNA laddering in cowpea leaf cells, but other toxic chemicals such as $\mathrm{CuSO}_{4}, \mathrm{ZnCl}_{2}$ and $\mathrm{NaN}_{3}$ did not induce laddering of nuclear DNA although they cause cell death [14]. NaCl at high concentration induces apoptosis of barley root cells [15]. More recently UV was shown to induce TUNEL stain and DNA cleavage in Arabidopsis seedlings and leaf protoplasts [16]. The UV dose required to induce PCD in Arabidopsis is 500-100 times higher than that used for animal cells, suggesting that plant cells may have a system to efficiently repair UV damage. It is increasingly clear that there exist many external stimuli which are able to induce plant cells, however whether any of the observed PCD are relevant to PCD observed during development and plant-microbe interaction remains to be studied.

\section{4. $\mathrm{PCD}$ as an integral component of disease resistance in plants}

Hypersensitive reaction (HR) has been observed in numerous plant species when they are attacked by avirlent pathogens including viruses, bacteria and fungi [17]. HR is characterized by localized cell death caused by pathogens and thought to restrict further invasion of pathogens. A current model of the signaling pathway involved in disease resistance is shown in Fig. 1. For instance, detailed cytological analysis of the interaction between rice and the avirulent blast fungus Magnaporthe grisea in leaf sheath cells indicates that the HR occurs at $17-25 \mathrm{hr}$ after inoculation, and is characterized by the appearance of granules in the cytoplasm, degeneration of nucleus and detachment of plasma membrane from the cell wall [18]. Rapid accumulation of reactive oxygen species (ROS) such as $\mathrm{O}_{2}{ }^{-}$and $\mathrm{H}_{2} \mathrm{O}_{2}$ has been observed at sites of infection and ROS are thought to trigger PCD. Close relationship between ROS production and PCD has been suggested by experiments in which leaves of the Arabidopsis cell death mutant, lsd 1, were treated with reagents that produce ROS [19]. The treatment induced cell death and it is inhibited by treatment with DPI, an inhibitor of NADPH oxidase. The results of these experiments and others sug-

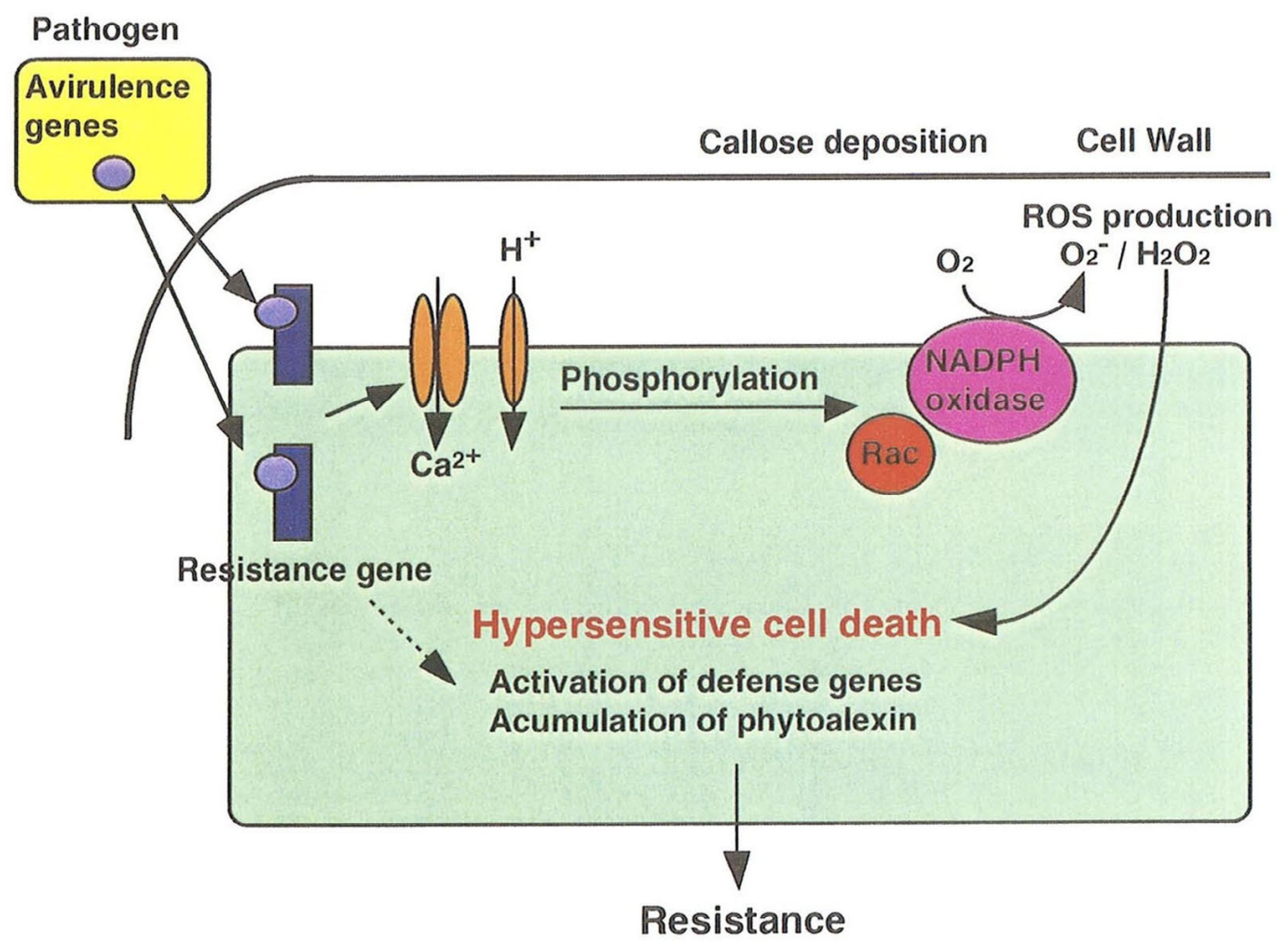

Fig. 1 A model of the signaling pathway for disease resistance in plants. Signals from pathogens are initially perceived by $R$ gene products. Then, the signal is transmitted through a protein phosphorylation cascade and activates NADPH oxidase at the plasma membrane, leading to ROS production. The Rac family of small GTP-binding protein regulates activation of the NADPH oxidase. ROS induce various downstream events including cell death. 


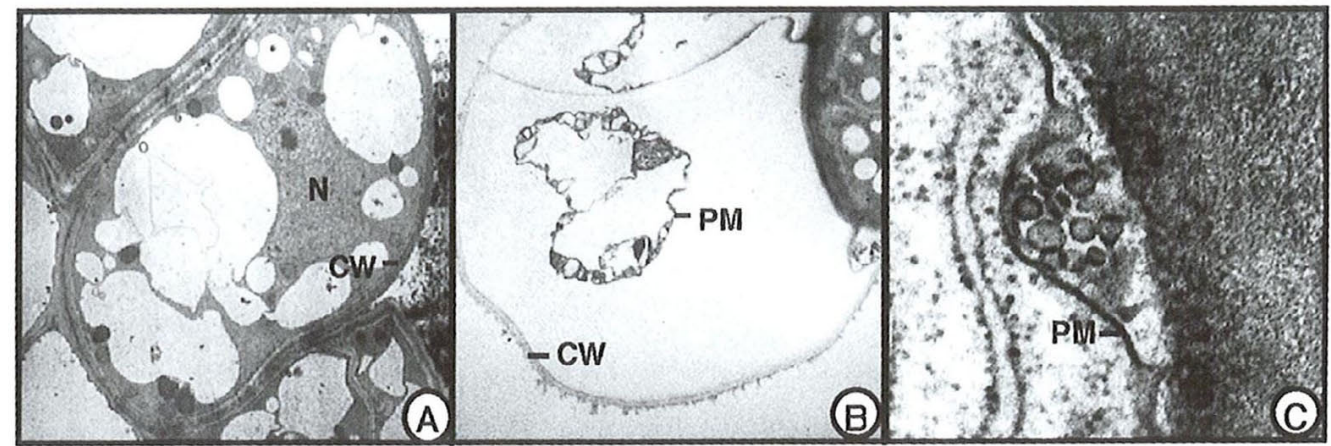

Fig. 2 Apoptosis-like cell death in OsRac-transformed rice cells in culture. (a) Untransformed control cell showing normal cell morphology. N: nucleus. CW: cell wall. (b) Shrinkage of plasma membrane in the OsRacl transformed cell. (c) Blebbing of plasma membrane in OsRacl-transformed cell. PM: plasma membrane.

gest involvement of NADPH oxidase in induction of PCD during plant-microbe interaction.

Since the NADPH oxidase may be a primary site of regulation leading to oxidative burst as well as PCD (Fig. 1), several groups have studied various aspects of this enzyme in plants. The mammalian NADPH oxidase is a complex consisting of 2 membrane-bound components, gp $91^{\text {phox }}$ and $\mathrm{p} 22^{\text {phox }}$, and 3 cytosolic components, p $47^{\text {phox }}, \mathrm{p} 67^{\text {phox }}$ and the small GTP-binding protein Rac2 [20]. Among them genes for homologs of gp 91 have been isolated from plants [21], however, their functional roles remain to be studied. We isolated 7 Rac-like genes of rice that have high amino acid identity with mammalian Rac and classified them into 4 groups based on their deduced amino acid sequences. They are termed $O s R a c$ and all were shown to be expressed in leaves and roots.

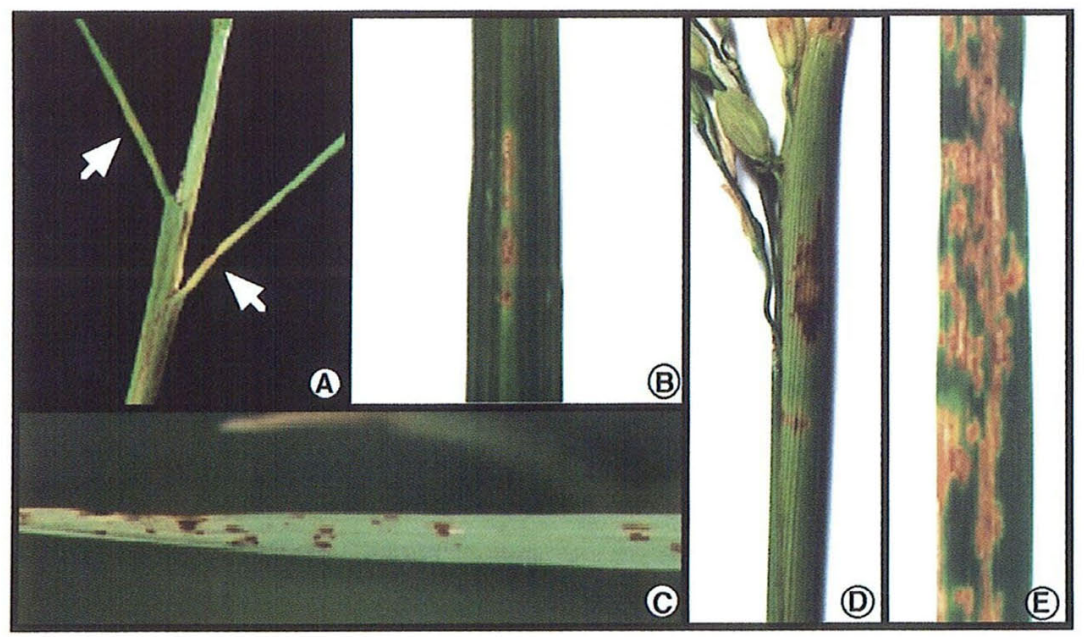

Fig. 3 Cell death in transgenic rice plants expressing the constitutively active form of OsRacl. (a-d) Cell death (lesions) found in leaves of transgenic wild type rice. (e) Cell death (lesions) found in transgenic lesion-mimic mutant of rice.
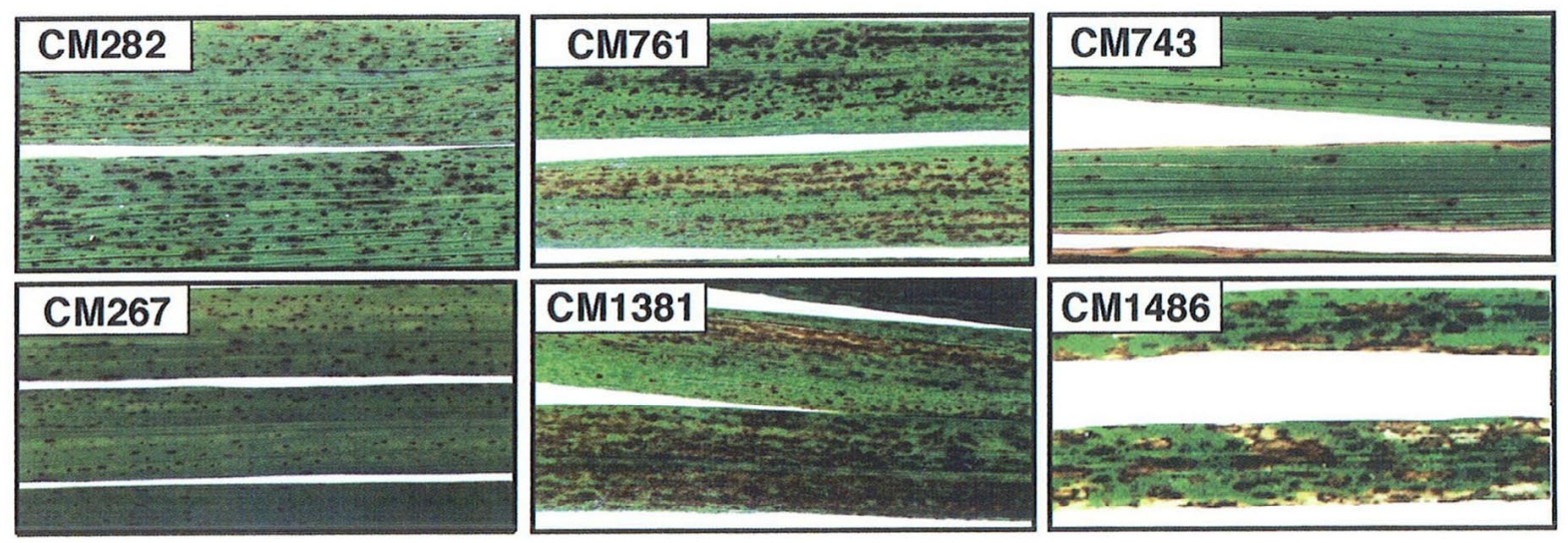

Fig. 4 Phenotypes of lesion-mimic mutants of rice. Lesions are variable with respect to the size, color and density. 
To elucidate possible roles of rice Rac genes in ROS production and PCD, we introduced constitutively-active and dominant-negative forms of OsRacl into rice and generated transgenic suspension cultures and plants [22]. When the constitutively-active form was introduced into a lesion-mimic mutant, its suspension culture showed the constitutive production of $\mathrm{H}_{2} \mathrm{O}_{2}$ and transformed cells exhibited morphological changes typical of animal cells undergoing apoptosis (Fig. 2). Furthermore, cell death was observed in leaves and stems of the transgenic plants produced from the mutant and wild type (Fig. 3). Furthermore, when the dominant-negative form was introduced into suspension cells $\mathrm{H}_{2} \mathrm{O}_{2}$ production caused by treatment with calyculin $\mathrm{A}$, an inhibitor of protein phosphatase, was suppressed. Development of lesions on the leaf of the lesion-mimic mutant was also inhibited by introduction of the dominant-negative form of OsRacl. These results strongly suggest that $\mathrm{Rac}$ is a regulator of ROS production as well as PCD in rice.

The preliminary analysis of transgenic rice expressing the constitutively active form of OsRacl indicates the transgenic plants showed HR when infected with the virulent race of the blast fungus (our unpublished results). Furthermore, spontaneous production of $\mathrm{H}_{2} \mathrm{O}_{2}$ and autofluorescence that are observed in plants showing resistance to the infection by the avirulent fungus are detected in the absence of the pathogen, further suggesting the close relationship of PCD and the disease resistance.

\section{Mutants showing spontaneous cell death}

Mutants that show spontaneous cell death in the absence of pathogens are called disease lesion mimics and have been long known in several cereals including maize, rice, and barley. They are either recessive or dominant; and almost all mutants each shows a unique lesion phenotype with respect to the size of the lesion, timing of lesion formation, and color of the lesion [23]. Mutant phenotypes are often influenced by environmental conditions such as light and temperature [23].

Systematic screening of similar mutants in Arabidopsis yielded a number of mutants called acd (accelerated cell death) and $l s d$ (lesion stimulating disease resistance) $[24,25]$. Detailed analysis of these mutants established that cell death is genetically programmed in plants. Furthemore, most of these mutants exhibited resistance to pathogens and concomitantly showed the typical HR frequently observed in plants challenged with avirulent pathogens. The molecular and cytological characteristics observed in these mutants are as follows: accumulation of autofluorescent material, callose deposition, activation of defense-related genes, elevated salycilic acid level, and activation of systemic aquired resistance pathway. These findings led to the conclusion that defense responses that are normally activated upon infection by avirulent pathogens are constitutively activated in this type of mutants without pathogens.

We have screened ca. 100 lesion mimic mutants of rice (Fig. 4) for resistance to blast fungus Magnaporthe grisea (anamorph, Pyricularia oryzae) and found 8 such mutants
[26]. Detailed analysis of 3 such mutants we called $c d r$ showed that a set of resistance responses are coordinately activated in these rice mutants. They show autofluorescence, callose deposition, accumulation of a phytoalexin, momilactone $\mathrm{A}$, and activation of defense-related genes, $P R-1$ and $P B Z-1$. Leaves of the $C d r 3$ mutant showed positive TUNEL staining indicating that apoptosis-like cell death occurs in the mutant. To elucidate possible biochemical alterations associated with these mutations, we produced suspension cultures from the mutants and analyzed $\mathrm{H}_{2} \mathrm{O}_{2}$ production induced by calyculin $\mathrm{A}$. Two mutants, $c d r l$ and $c d r 2$, showed enhanced $\mathrm{H}_{2} \mathrm{O}_{2}$ production while $C d r 3$ exhibited the similar level of $\mathrm{H}_{2} \mathrm{O}_{2}$ production as wild type. These results suggest that $c d r 1$ and $c d r 2$ may have alterations in the signal transduction pathway leading to activation of the NADPH oxidase and $C d r 3$ may have an alteration downstream of the NADPH oxidase (Fig. 5).

\section{Future prospects}

Recent progress in research on PCD in plants clearly indicates that PCD occurs in various organs during de-

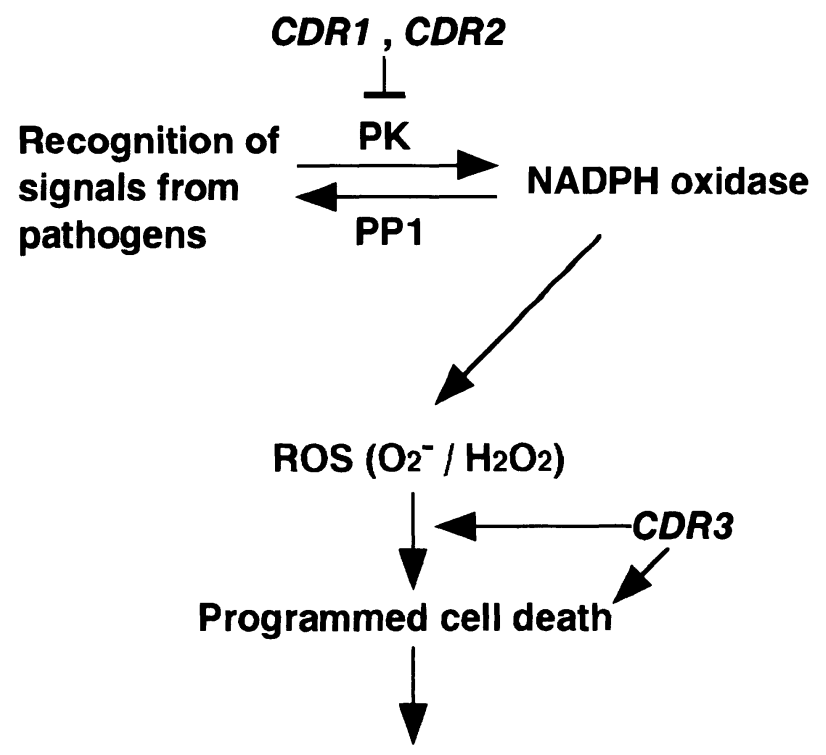

Accumulation of autofluorescent material Expression of defense-related genes

Accumulation of phytoalexin

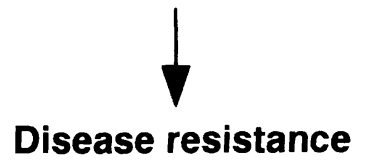

Fig. 5 A model for possible biochemical functions of the genes encoded by three lesion-mimic mutants of rice, $c d r l, c d r 2$ and Cdr3. This model is based on the analysis of $\mathrm{H}_{2} \mathrm{O}_{2}$ production in cultured mutant cells in response to protein phosphatase inhibitors. The $c d r 1$ and $c d r 2$ mutations are in the genes that normally suppress functions of protein kinases involved in activation of the NADPH oxidase. In contrast, $C d r 3$ may encode a factor functioning downstream of the NADPH oxidase. Since the mutation is dominant, the wild type gene may encode either activator or suppressor. PK: protein kinase. PP1: protein phosphatase 1. 
velopment and that it plays major roles in the resistance responses to avirulent pathogens. Recent studies demonstrate that plant PCD is associated with cellular and biochemical changes characteristic of apoptosis in mammalian cells; TUNEL staining, DNA laddering, and cell shrinkage and membrane blebbing have been observed in multiple systems in plants. Thus it is clear now that PCD in plants shares many, if not all, features with apoptosis in animals although more detailed studies are required to better define plant PCD at cellular and biochemical levels.

Although occurrence of PCD in plants is recently becoming clear little is known for its molecular mechanisms. Enzymes such as protease and DNase that are possibly involved in PCD have been described in plants. More recently existence of caspases in plants is suggested by studies using inhibitors. Roles of these enzymes in PCD remain to be determined. Our studies suggest that the small GTP-binding protein Rac plays a role in triggering PCD in plants. Therefore, the future research on plant PCD should focus on identification of signaling molecules involved in induction of $\mathrm{PCD}$ and of components of machinery operative in various types of PCD in plants.

\section{Acknowledgements}

We thank Drs K. Shii and O. Kodama of Ibaraki University and Dr. H. Satoh of Kyushu University for collaborations on our research on rice PCD. We also thank the members of Laboratory of Plant Genetics for discussions.

\section{References}

[1] Greenberg, J.T., 1996. Proc. Natl. Acad. Sci. USA, 93: 12094-12097.

[2] Dangl, J.L., Dietrich, R.A., Richberg, M.H., 1996. Plant Cell, 8: 1793-1807.

[3] Pennell, R.I., Lamb, C., 1997. Plant Cell, 9: 1157-1168.

[4] Ashkenazi, A., Dixit, V.M., 1998. Science, 281: 1305-1308.
[5] Thornberry, N.A., Lazebnik, Y., 1998. Science, 281: 13121316.

[6] Wong, M., Oppedijk, B.J., Lu, X., Van Duijn, V., Schilperoort, R.A., 1996. Plant Mol. Biol., 32: 1125-1134.

[7] Fukuda, H., 1997. Plant Cell, 9: 1147-1156.

[8] He, C.-J., Morgan, P.W., Drew, M.C., 1996. Plant Physiol., 112: 463-472.

[9] Wang, H., Li, J., Bostock, R.M., Gilchrist, D.G., 1996. Plant Cell, 8: 375-391.

[10] Smart, C.M., 1994. New Phytol., 126: 419-448.

[11] Orzaez, D., Granell, A., 1997. Plant J., 11: 137-144.

[12] Dellaporta, S.L., Calderon-Urrea, A., 1993. Plant Cell, 5: 1241-1251.

[13] Levine, A., Pennell, R.I., Alvarez, M.E., Palmer, R., Lamb, C., 1996. Curr. Biol., 6: 427-437.

[14] Ryerson, D.E., Heath, M.C., 1996. Plant Cell, 8: 393-402.

[15] Katsuhara, M., 1997. Plant Cell Physiol., 38: 1091-1093.

[16] Danon, A., Gallois, P., 1998. FEBS Lett., 437: 131-136.

[17] Hammond-Kosack, K.E., Jones, J.D.G., 1996. Plant Cell, 8: 1773-1791.

[18] Koga, H., 1994. Can. J. Bot., 72: 1463-1477.

[19] Jabs, T., Dietrich, R.A., Dangl, J.L., 1996. Science, 273: 1853-1856.

[20] Segal, A.W., Abo, A., 1993. Trends in Biochem. Sci., 18: 43-47.

[21] Keller, T., Damude, H.G., Werner, D., Doerner, P., Dixon, R.A., Lamb, C., 1998. Plant Cell, 10: 255-266.

[22] Henmi, K., Kawasaki, T., Hatakeyama, S., Iwano, M., Satoh, H., Shimamoto, K., submitted.

[23] Johal, G.S., Hulbert, S.H., Briggs, S.P., 1995. Bioessays, 17: $685-692$.

[24] Greenberg, J.T., Guo, A., Klessig, D.F., Ausubel, F.M., 1994. Cell, 77: 551-563.

[25] Dietrich, R.A., Delaney, T.P., Uknes, S.J., Ward, E.R., Ryals, J.A., Dangl, J.L., 1994. Cell, 77: 565-577.

[26] Takahashi, A., Kawasaki, T., Henmi, K., Shii, K., Kodama, O., Satoh, H., Shimamoto, K., 1999. Plant J., in press. 